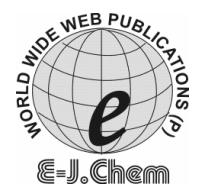

http://www.e-journals.net
ISSN: 0973-4945; CODEN ECJHAO

E-Journal of Chemistry 2010, 7(3), 870-874

\title{
DFT Modelling of Tripeptides (Lysine-Tryptophan-Lysine) Interacting with Single Walled Carbon Nanotubes
}

\author{
NAVARATNARAJAH KUGANATHAN \\ Department of Chemistry, \\ University of Bath, Bath, BA2 7AY, England, UK. \\ nk247@bath.ac.uk
}

Received 17 August 2009; Accepted 10 October 2009

\begin{abstract}
Model calculations are performed to predict the nature of interaction between SWNT and a tripeptide (Lys-Trp-Lys) and to calculate the binding energies and charge transfer between these two species using density functional theory. DFT calculations indicate that the interaction is of a non covalent nature. Minimal charge transfer is observed between SWNT and LysTrp-Lys.
\end{abstract}

Keywords: Carbon nanotubes, DFT, Tripeptide, Binding energy, Charge transfer.

\section{Introduction}

Carbon nanotubes are hollow graphitic nanomaterials with outer diameters of 4-30 nm and lengths up to $1 \mu \mathrm{m}^{1}$. Single-walled carbon nanotubes (SWNTs) which are made of a single graphene sheet were first reported in 1993 by Iijima et.al. ${ }^{2}$ SWNTs are simpler in structure and have a smaller range of diameters compared with MWNTs. In the absence of interactions between neighbouring tubes walls found in MWNTs, it is easier to predict physical properties of SWNTs, using computer calculations and theoretical models.

Functionalisation of tripeptides with SWNTs is of fundamental importance for the use of SWNTs in biosensing, biocompatibility or delivery applications ${ }^{3}$. Polypeptides adsorbed on to SWNTs has been used to prepare SWNT composite materials. The curved $\pi$ surface of SWNT shows a tendency to interact with polypeptides. Recently Christoph et al. have reported the interaction of tryptophan (Trp) and lysine (Lys) containing polypeptides with SWNTs $^{4}$. In this study, a considerable interaction has been observed between SWNT and copolymers containing tryptophan and lysine. As the exact structure of SWNTs intercalated in tripeptide receptors is not known, we modelled a simple tripeptide structure containing tryptophan and lysine and a capped [10,10] SWNT. Recently, DFT-based codes, such as 
SIESTA $^{5}$, which allow for $a b$ initio modelling of large systems, have been developed. In our previous study, we have successfully modelled a host-guest composite containing SWNT and a tripodal porphyrin host to investigate the nature of interaction ${ }^{6}$. No reports of DFT based studies on the interactions between SWNTs and Lys-Trp-Lys are available. Therefore, in this article we model the structure of SWNT interacting with a tripeptide (Lys-Trp-Lys) and perform DFT calculations to evaluate the binding nature and charge transfer between these two species.

\section{Computational Methods}

Electronic structure calculations were performed on tripeptide interacting with SWNT using DFT methods. All calculations were performed using the SIESTA code ${ }^{7,8}$. The exchangecorrelation term was modelled using the local density approximation (LDA) parameterized by Ceperley and Alder ${ }^{9}$. Standard norm conserving Troullier-Martins pseudopotentials were used to model the core electrons of $\mathrm{C}, \mathrm{N}, \mathrm{O}$ and $\mathrm{H}^{10-12}$. The reference electronic configuration, cut-off radius and partial core cut-off radius for all pseudopotentials employed here are tabulated (Table 1). For N, a partial core correction is necessary to account for nonlinearity of the exchange and correlation potential between core and valence charge densities.

Table 1. Reference configuration and cut-off radii (a.u.) of the pseudopotentials used in this study.

\begin{tabular}{lccccc}
\hline \multicolumn{1}{c}{ Atom } & $\mathbf{C}$ & $\mathbf{N}$ & $\mathbf{O}$ & $\mathbf{H}$ \\
\hline Reference & $2 s^{2} 2 p^{2} 3 d^{0} 4 f^{0}$ & $2 s^{2} 2 p^{3} 3 d^{0} 4 f^{0}$ & $2 s^{2} 2 p^{4} 3 d^{0} 4 f^{0}$ & $1 s^{2} 2 p^{0} 3 d^{0} 4 f^{0}$ \\
Core radius (a.u.) & $s$ & 1.25 & 1.25 & 1.15 & 1.25 \\
& $p$ & 1.25 & 1.25 & 1.15 & 1.25 \\
& $d$ & 1.25 & 1.25 & 1.15 & 1.25 \\
& $f$ & 1.25 & 1.25 & 1.48 & 1.25 \\
Core cutoff (a.u.) & & 0.00 & 1.37 & 0.00 & 0.00 \\
\hline
\end{tabular}

To test the quality of the pseudopotential, comparisons were made with the eigen values and excitation energies of all electron calculations on the same series of atomic configurations. The cross-excitation energies for both $\mathrm{C}$ and $\mathrm{N}$ pseudo potentials were not more than $0.09 \mathrm{eV}$, for $\mathrm{O}$ and $\mathrm{H} 0.05 \mathrm{eV}$, indicating the excellent transferability of these pseudo potentials. The one-electron Kohn-Sham eigenstates were expanded in a basis of strictly localized numerical atomic orbitals ${ }^{13}$. For carbon, nitrogen and oxygen, a double- $\zeta$ basis set for $2 s$ and $2 p$ valence states and a single- $\zeta$ basis set for $3 d$ were used. For $\mathrm{H}$ a double- $\zeta$ basis set for $1 s$ and a single- $\zeta$ basis set for the $2 p$ orbitals were used.

Structure optimizations were performed using a conjugate gradient algorithm and the forces on the atoms were obtained from the Hellman-Feynmann theorem including Pulay corrections. In all optimised structures forces on the atoms were smaller than $0.06 \mathrm{eV} / \AA$ and the stress tensor was less than $0.02 \mathrm{GPa}$.

To represent the charge density a cut-off of 200 Ry for the real space grid integration was used in all calculations. We have used super cells with 40 A vacuum spaces along $\mathrm{x}, \mathrm{y}$ and $\mathrm{z}$ directions for SWNTs wrapped with tripeptides. A single k point $(\Gamma)$ was used for all calculations.

\section{Molecular modelling}

Density functional calculations were used to understand the geometry adopted by the tripeptide in the presence of SWNTs and the nature of the interactions between the tripeptide 
and SWNTs. In this paper, we propose a new models for the gas-phase structures of composites which can be formed between a tripeptide and an idealised, capped, SWNT.

To model the structure of the composite, formed between tripeptides and SWNTs, a capped $(10,10)$ SWNT and a single tripeptide unit were used. Figure 1 show the tripeptide [Lysine-Tryptophan-Lysine (Lys-Trp-Lys)] used in this modelling.

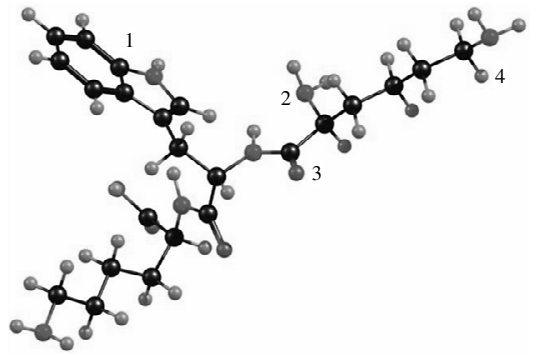

Figure 1. Structure of lysine-tryptophan-lysine (Lys-Trp-Lys) (Black (1) - Carbon, Pink (2)- Nitrogen, Red (3) - Oxygen, Blue (4)- hydrogen)

\section{Results and Discussion}

The optimised structure suggests that Lys-Trp-Lys is able to interact with different orientations. The optimised structures are given in Figure 2. The closest $\mathrm{C}-\mathrm{C}$ distances between Lys-Trp-Lys and SWNT are tabulated in Table 2.

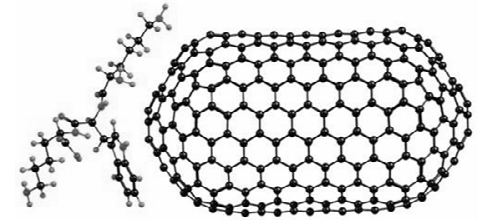

Model 1

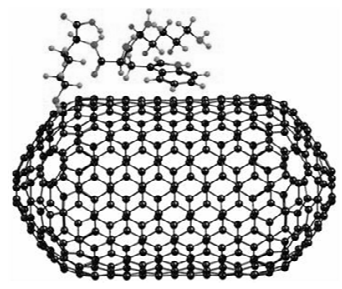

Model 2

Figure 2. DFT-level optimised structures of two different models of composites.

Table 2. Closest C-C distances between SWNT and tripeptides.

\begin{tabular}{cc}
\hline Type & Closest C-C distances between SWNT and Lys-Trp-Lys $(\AA)$ \\
\hline Model 1 & $3.08,3.15,3.20,3.23,3.30,3.42$ \\
Model 2 & $3.20,3.24,3.33,3.37$ \\
\hline
\end{tabular}

The closest C-C distances indicate that there are no strong bonds between SWNT and Lys-Trp-Lys and the interaction is based on $\pi-\pi$ type. The binding energy between the tripeptide and SWNT would be ideally described by the difference between the total energy of the SWNT_TP (TP- Tripeptide) composite and the total energies calculated for an isolated SWNT unit and an isolated tripeptide. Subtraction of the energy quantities of both SWNT and TP from the composite with localized basis sets gives an uncorrected binding energy, $E_{b(\text { unc })}$, as defined by equation (1)

$$
E_{b(\text { unc })}=E_{S W N T}-T P^{-} E_{S W N T}-E_{T P}
$$

Where $E_{S W N T_{-} T P}$ is the total energy of SWNT complexed by the host (TP) and $E_{S W N T}$ and $E_{T P}$ are the total energies of isolated SWNT and host (TP) respectively. The error in binding energy due to Basis Set Superposition Errors (BSSE) can be high. Therefore, this BSSE 
must be corrected in DFT calculations employing localized basis sets. The true binding energy can be determined by the counterpoise correction method of Boys and Bernardi ${ }^{14}$ using "ghost" atoms. The "ghost" molecule corresponds to additional basis wave functions centered at the atomic positions of SWNT or tripeptide, but without any atomic potential. The binding energies (Table 3) indicate that the interaction is non covalent. According to the calculated binding energies, it is clear that the interaction between SWNT as a tripeptide is of non-covalent nature.

Table 3. Uncorrected binding energies, $E_{b(u n c)}(\mathrm{eV})$ and counterpoise corrected binding energies, $E_{b(C P)}(\mathrm{eV})$.

\begin{tabular}{ccc}
\hline Type & \multicolumn{2}{c}{ Lys-Trp-Lys } \\
\hline & $E_{\boldsymbol{b}(\boldsymbol{u n n})}$ & $E_{\boldsymbol{b}(\boldsymbol{C P})}$ \\
Model 1 & -0.88 & -0.66 \\
Model 2 & -1.11 & -0.68 \\
\hline
\end{tabular}

One of the limitations in DFT is its overestimation of the binding energy due to weak interactions ${ }^{15}$. This problem has been highlighted by performing calculations on a benzene dimer. DFT calculations yield an inter-ring distance of $3.06 \AA$ and a binding energy of -0.48 $\mathrm{eV}^{15,16}$. However higher level CCSD $(\mathrm{T})$ calculation values are considerably different and are estimated to be $4.1 \AA$ and $-0.05 \mathrm{eV}^{16}$.

The shortest C-C distances between the tripeptide unit and SWNT ranged between 3.08 and $3.52 \AA$ in both cases. Unfortunately, X-ray structural data for the SWNT@tripeptide is unavailable to compare these values with experiment. However, in our previous study, we have modelled and performed calculations on porphyrin @ $\mathrm{C}_{60}$ and in this case DFT gave results which are in excellent agreement with experiment and also it validated our computational approach ${ }^{6}$.

A negligible charge transfer is observed between SWNT and tripeptide. For the SWNT: Lys-Trp-Lys complex optimised, the estimated charge transfer value from tripeptide to SWNT was of 0.030 electrons in model 1 and 0.046 in model 2. Table 4 summarises the amount of charge transferring from tripeptide to nanotube.

Table 4. Charges calculated for the interaction of tripeptide with SWNT.

\begin{tabular}{cc}
\hline Type & Charge Transfer [(Lys-Trp-Lys) $\rightarrow$ SWNT] \\
\hline Model 1 & 0.030 \\
Model 2 & 0.046 \\
\hline
\end{tabular}

\section{Conclusion}

In conclusion, gas phase DFT modelling was performed to see the effect of the interaction between SWNT and a tripeptide. The optimised structure of tripeptide@SWNT with an exothermic binding energy indicates that the host is able to interact with a guest with a wider diameter such as $1.8 \mathrm{~nm}$ (i.e. a $[10,10] \mathrm{SWNT})$. The interaction is of a non-covalent nature. Negligible charge transfer is observed between tripeptide and SWNT.

\section{References}

1. Iijima S, Nature, 1991, 354, 56 .

2. Iijima S and Ichihashi T, Nature, 1993, 363, 603.

3. Bianco A and Prato M, Adv Mater., 2003, 15, 1765. 
4. Salzmann C G, Ward M A H, Jacobs R M J, Tobias G, Green and M L H, J Phys Chem C., 2007, 111, 18520.

5. Ordejon P, Sanchez-Portal D, Garcia A, Artacho E, Junquera J and Soler J M, RIKEN Review, 2000, 29, 42.

6. Pascu S I, Kuganathan N, Tong L H, Jacobs R M, Chu B T, Salzmann C G, Tobias G, Ward M, Green J C, Green M L H and Sanders J K M, J Mater Chem., 2008, 18, 2781.

7 Artacho E, Sanchez-Portal D, Ordejon P, Garcia A and Soler J M, Phys Status Solidi B: Basic Research., 1999, 215, 809.

8. Soler J M, Artacho E, Gale J D, Garcia A, Junquera J, Ordejon P and Sanchez-Portal D, J Phys Condens Mater., 2002, 14, 2745.

9. Ceperley D M and Alder B J, Phys Rev Lett., 1980, 45, 566.

10. Bachelet G B and Schlueter M, Phys Rev B., 1982, 25, 2103.

11. Fuchs M and Scheffler M, Comput Phys Commun., 1999, 119, 67.

12. Troullier N and Martins J L, Phys Rev B., 1991, 43, 1993.

13. Junquera J, Paz O, Sanchez-Portal D and Artacho E, Phys Rev B., 2001, 64, 235111.

14. Boys S F and Bernardi F, Mol Phys., 1970, 19, 553.

15. Sceats E L and Green J C, J Chem Phys., 2006, 125, 154704

16. Hobza P, Selzle H L and Schlag E W, J Phys Chem., 1996, 100, 18790. 


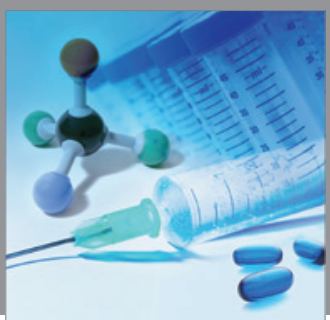

International Journal of

Medicinal Chemistry

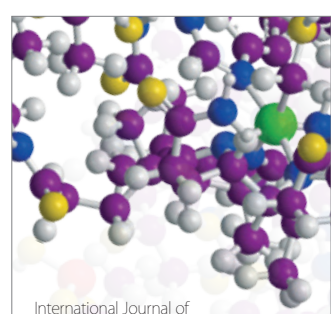

Carbohydrate Chemistry

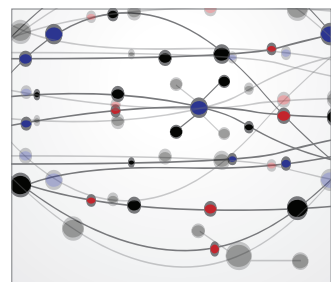

The Scientific World Journal
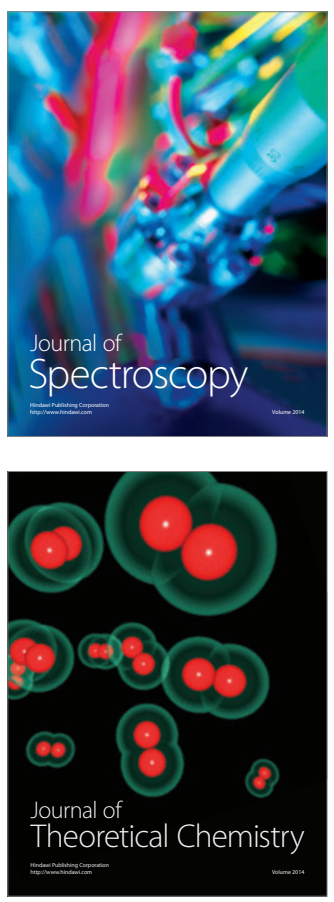
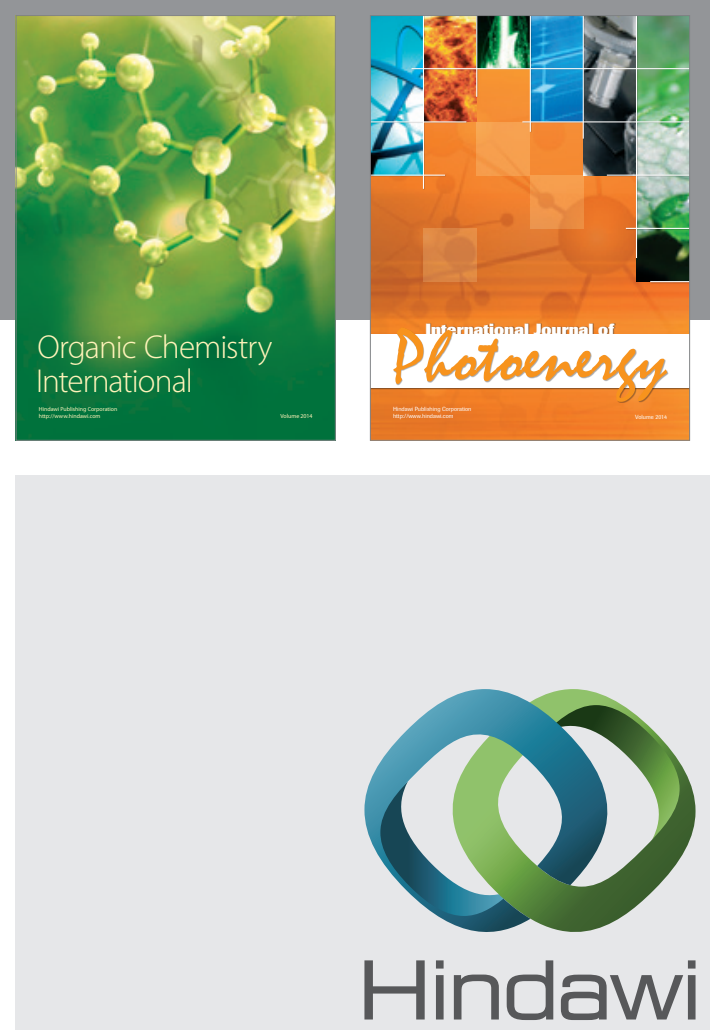

Submit your manuscripts at

http://www.hindawi.com
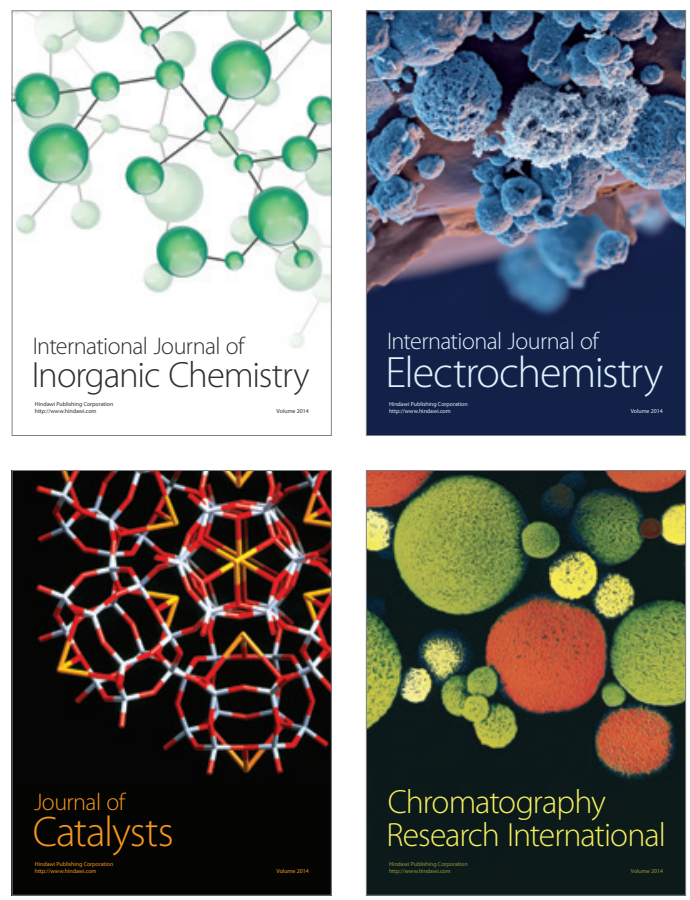
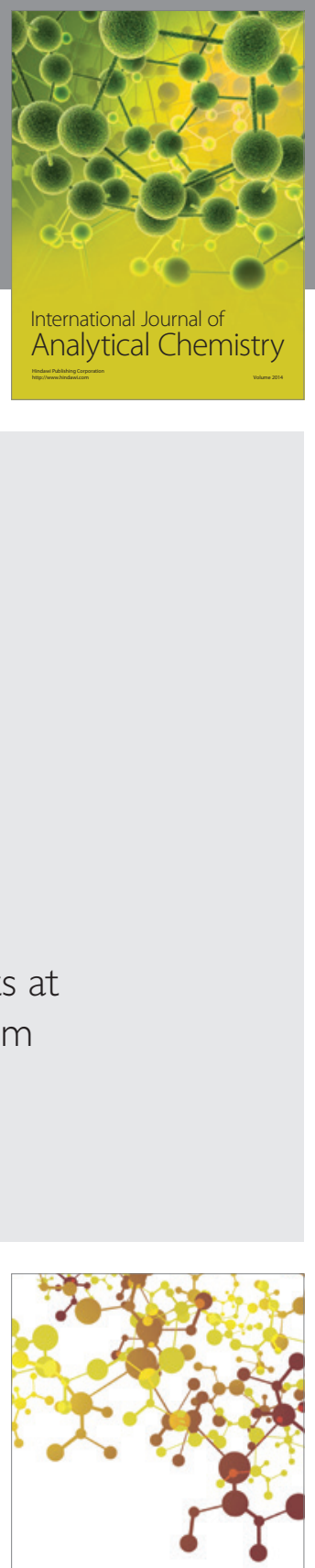

Journal of

Applied Chemistry
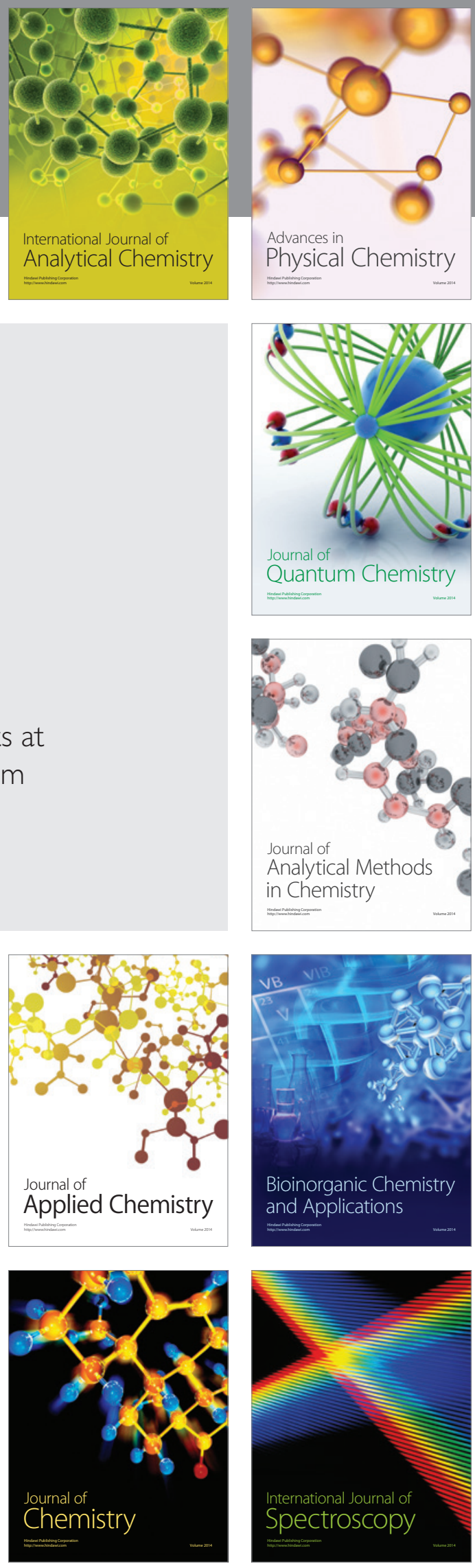